\title{
A Study on New Muller's Method
}

\author{
By \\ Bong-kyu PARK* and Sin Hitotumatu**
}

\begin{abstract}
When we compute a root of equation $F(X)=0$, Muller's Method uses three initial approximations $X_{0}, X_{1}$, and $X_{2}$ and determines the next approximation $X_{3}$ by the intersection of the $\mathrm{X}$-axis with the parabola through $\left(X_{0}, F\left(X_{0}\right)\right),\left(X_{1}, F\left(X_{1}\right)\right)$, and $\left(X_{2}, F\left(X_{2}\right)\right)$. The procedure is repeated successively to improve the approximate solution of an equation $F(X)$ $=0$.

Suppose a continuous function $F$, defined on the interval $\left[X_{0}, X_{1}\right]$ is given, with $F\left(X_{0}\right)$ and $F\left(X_{1}\right)$ being opposite signs. In our New-Muller's Method we choose $X_{0}$ and $X_{1}$ as the ends of the interval and take another initial approximation $X_{2}$ as the mid-point of $X_{0}, X_{1}$ and new approximation $X_{3}$ is the intersection of the $\mathrm{X}$-axis with a quadratic curve through $\left(X_{0}, F\left(X_{0}\right)\right),\left(X_{2}, F\left(X_{2}\right)\right)$, and $\left(X_{1}, F\left(X_{1}\right)\right)$. This method is proposed to improve the rate of convergence and calculate faster for reducing the interval. Let us call this method NewMuller's Method in this paper.
\end{abstract}

\section{§1. New-Muller's Method}

Let $F(X)$ be continuous function that has a root in an interval $\left[X_{0}, X_{1}\right]$.

Beginning with the initial approximations $X_{0}$ and $X_{1}$ under the condition $F\left(X_{0}\right) \cdot F\left(X_{1}\right)<0$ an intermediate initial approximation is taken as $X_{2}=\left(X_{0}+X_{1}\right) / 2$.

Then $X_{3}$ is the intersection of the $\mathrm{X}$-axis with a quadratic curve $G(X)=0$ through three point $\left(X_{0}, F\left(X_{0}\right)\right),\left(X_{2}, F\left(X_{2}\right)\right)$, and $\left(X_{1}\right.$, $F\left(X_{1}\right)$ ).

Next we determine the closed interval $I$ which includes the solution of $F(X)=0$, as follow;

1) If $F\left(X_{3}\right) F\left(X_{0}\right)<0 \Longrightarrow I_{0}=\left[X_{0}, X_{3}\right]$

1) If $F\left(X_{3}\right) F\left(X_{1}\right)<0 \Longrightarrow I_{0}=\left[X_{3}, X_{1}\right]$

Communicated by H. Araki, February 17, 1987. Revised April 13, 1987.

* Yuhan Technical College, Seoul Korea.

** Research Institute for Mathematical Sciences, Kyoto University, Japan. 
1) If $F\left(X_{3}\right) F\left(X_{2}\right)<0$
a) $X_{3}<X_{2} \Longrightarrow I_{0}=\left[X_{3}, X_{2}\right]$
b) $X_{3}>X_{2} \quad \Longrightarrow I_{0}=\left[X_{2}, X_{3}\right]$

The closed interval $I_{0}$ is determined as above. Note that the length of $I_{0}$ is diminished less than half of the original interval in any case. To find the second approximation, we put newly the lower bound of $I_{0}$ as $X_{0}$, and upper bound of $I_{0}$ as $X_{1}$. We repeat above New-Muller's Method to find the nearer root of the quadratic equation whose curve passes through the last three points, and this root of the quadratic equation is included in closed interval. Now we select a contracted closed interval $I$ described as above.

We continue the process and have a nested closed intervals $I_{0}, I_{1} \ldots$ We repeat it until the interval shrinks sufficiently near the solution.

Definition 1-1) We take $X_{0}$ and $X_{1}$ such that $F\left(X_{0}\right)$ and $F\left(X_{1}\right)$ have the opposite signs. $X_{2}$ is another intermediate initial approximation determined by the initial approximations $X_{0}, X_{1}$; that is, $X_{2}=$ $\left(X_{0}+X_{1}\right) / 2$ 。

Definition 1-2) The closed interval determined by New-Muller's Method is called New-Muller's restricted closed interval.

Although there may occur several cases according to the signatures of $F\left(X_{n}\right)$, it is not difficult to show that the following theorem holds in any cases.

Theorem 1-1) Let $F(X)$ be continuous on the closed interval $\left[X_{n}, X_{n+1}\right]$. We apply the above procedure to have the sequence of intervals $I_{n}$, where $n=0,1,2, \ldots$ Then $\mathbb{N e w}$-Muller's Method gives a Cantor's sequence of the nested closed interval, and if $n \rightarrow \infty$ then $I_{n} \rightarrow R . \quad(R$ is a root of $F(X)=0$ 。)

\section{§ 2. Method of Calculation}

When the initial approximations $X_{0}$ and $X_{1}$ satisfies $F\left(X_{0}\right) \cdot F\left(X_{1}\right)$ $<0$ another initial value is $X_{2}=\left(X_{0}+X_{1}\right) / 2$. Let us put the quadratic polynomial that passes through the three points $\left(X_{0}, F\left(X_{0}\right)\right)$ $\left(X_{2}, F\left(X_{2}\right)\right)$, and $\left(X_{1}, F\left(X_{1}\right)\right)$ to be 


$$
G(X)=a\left(X-X_{1}\right)^{2}+b\left(X-X_{1}\right)+c 。
$$

From the above conditions we have

$$
\begin{aligned}
& F\left(X_{0}\right)=a\left(X_{0}-X_{1}\right)^{2}+b\left(X_{0}-X_{1}\right)+c \\
& F\left(X_{2}\right)=a\left(X_{2}-X_{1}\right)^{2}+b\left(X_{2}-X_{1}\right)+c \\
& F\left(X_{1}\right)=a 0+b 0+c
\end{aligned}
$$

and we obtain

$$
\begin{aligned}
& a=\frac{2\left[F\left(X_{0}\right)-F\left(X_{1}\right)\right]-4\left[F\left(X_{2}\right)-F\left(X_{1}\right)\right]}{\left(X_{0}-X_{1}\right)^{2}} \\
& b=\frac{4\left[F\left(X_{2}\right)-F\left(X_{1}\right)\right]-\left[F\left(X_{0}\right)-F\left(X_{1}\right)\right]}{X_{0}-X_{1}} \\
& c=F\left(X_{1}\right)
\end{aligned}
$$

To find out the root $X$ which is $G(X)=0$, we use the formular for the quadratic equation. We modify the formular as follows to avoid the error arised by subtracting two close numbers:

$$
X_{3}=X_{1}-2 c /\left(b+\operatorname{sign}(b)\left(b^{2}-4 a c\right)^{1 / 2}\right)
$$

Here $\operatorname{sign}(b)$ is determined by the same method as Muller's Method.

Next initial approximation $X$ are obtained as described in $\S 1$. This process is repeated continuously until a satisfactory solution is found.

\section{§3. New-Muller's Algorithm}

Suppose a continuous function $F$ defined in the interval $\left[X_{0}, X_{1}\right]$, is given with $F\left(X_{0}\right)$ and $F\left(X_{1}\right)$ being opposite signs. This producess uses following algorithm.

Step 1 set $i=1$

Step 2 while $i \leq N$ do step 3-9

Step 3 set $X_{2}=\left(X_{0}+X_{1}\right) / 2$ (compute $X$ )

Step 4 If $F\left(X_{2}\right)=0$ or $\left(X_{1}-X_{0}\right) / 2<$ EPS then

OUTPUT $\left(X_{2}\right)$ : (Procedure completed successfully) STOP

Step 5 set $h=X_{1}-X_{0}$;

$$
\begin{aligned}
& S=F\left(X_{0}\right)-F\left(X_{1}\right) ; \\
& S=F\left(X_{2}\right)-F\left(X_{1}\right) ; \\
& a=\left(2 S_{1}-4 S_{2}\right) / h ; \\
& b=\left(4 S_{2}-S_{1}\right) / h ;
\end{aligned}
$$




$$
c=F\left(X_{1}\right)
$$

Step $6 D=(b-4 a c)^{1 / 2}$

Step 7 If $|b-D|<|b+D|$ then set $E=b+D$

else set $E=b-D$

Step 8 Set $h 1=-2 c / E$

$$
X_{3}=X_{2}+h
$$

Step 9 If $|h|<\operatorname{EPS}$ then

OUTPUT $\left(X_{3}\right)$; (Procedure completed successfully)

STOP

Stepl0 If $F\left(X_{2}\right) F\left(X_{0}\right)<0$

then set $X_{1}=X_{2}$ GO TO Step 2

Stepl1 If $F\left(X_{3}\right) F\left(X_{2}\right)<0$ and $X_{2}<X_{3}$

then set $X_{0}=X_{2}, X_{1}=X_{3}$ GO TO Step 2

Step12 If $F\left(X_{3}\right) F\left(X_{1}\right)<0$

then set $X_{0}=X_{3}$ GO TO Step 2

Stepl3 If $F\left(X_{3}\right) F\left(X_{0}\right)<0$

then set $X_{1}=X_{3}$ GO TO Step 2

Step14 If $F\left(X_{3}\right) F\left(X_{2}\right)<0$ and $X_{3}<X_{2}$

then set $X_{0}=X_{3}, X_{1}=X_{2}$ GO TO Step 2

Stepl5 If $F\left(X_{2}\right) F\left(X_{3}\right)<0$

then set $X_{0}=X_{2}$ GO TO Step 2

\section{Examples;}

1) $X^{3}-X-1=0$

2) $X^{4}-3 X^{3}-X^{2}+2 X+3=0$

3) $X^{5}-2 X^{4}-4 X^{3}+X^{2}+5 X+3=0$

4) $X^{6}-8 X^{4}-4 X^{3}+7 X^{2}+13 X+6=0$

5) $X^{7}+X^{6}-8 X^{5}-12 X^{4}+3 X^{3}+20 X^{2}+19 X+6=0$

The results are as follows by Muller's Method and New Muller's

\begin{tabular}{|c|c|c|c|c|}
\hline \multirow{2}{*}{$\begin{array}{c}\text { Degree of } \\
\text { Equation }\end{array}$} & \multicolumn{2}{|c|}{$\begin{array}{c}\text { Accuracy of last } \\
\text { Root to be found }\end{array}$} & \multicolumn{2}{c|}{ Times of Repeating } \\
\cline { 2 - 5 } & Muller & New Muller & Muller & New Muller \\
\hline 3 & $10^{-12}$ & $10^{-12}$ & 6 & 4 \\
4 & $10^{-12}$ & $10^{-12}$ & 5 & 5 \\
5 & $10^{-12}$ & $10^{-12}$ & 6 & 5 \\
6 & $10^{-12}$ & $10^{-12}$ & 6 & 4 \\
\hline
\end{tabular}

(Table a) 
Method for above.

We have failed in being computed by Muller's Method as below Table b. (This Table $b$ is a case of Example 5).)

\begin{tabular}{|c|c|}
\hline Solved case with needed root & failed case \\
\hline RUN-NUMBER $=20$ & RUN-NUMBER $=20$ \\
$\mathrm{~S}(1)=1.000$ & $\mathrm{~S}(1)=1.000$ \\
$\mathrm{~S}(2)=1.000$ & $\mathrm{~S}(2)=1.000$ \\
$\mathrm{~S}(3)=-8.000$ & $\mathrm{~S}(3)=-8.000$ \\
$\mathrm{~S}(4)=-12.000$ & $\mathrm{~S}(4)=-12.000$ \\
$\mathrm{~S}(5)=3.000$ & $\mathrm{~S}(5)=3.000$ \\
$\mathrm{~S}(6)=20.000$ & $\mathrm{~S}(7)=19.000$ \\
$\mathrm{~S}(7)=19.000$ & $\mathrm{~S}(8)=6.000$ \\
$\mathrm{~S}(8)=6.000$ & $\mathrm{X} 0=0 . \quad \mathrm{X} 1=0.50000000000000$ \\
$\mathrm{X} 0=1.5000000000000$ & $\mathrm{X} 2=1.0000000000000$ \\
$\mathrm{X} 1=2.0000000000000$ & $\mathrm{XANS}=-0.181953492716743103763121781$ \\
$\mathrm{X} 2=2.5000000000000$ & $\mathrm{XANS}=-0.297688107790282336928555651$ \\
$\mathrm{XANS}=1.486557539197503663430666165$ & $\mathrm{XANS}=-0.595205607486539223227595130$ \\
$\mathrm{XANS}=1.480369343312102958787335183$ & $\mathrm{XANS}=-0.812730423617922159706949969$ \\
$\mathrm{XANS}=1.475050097625060563366616861$ & $\mathrm{XANS}=-0.680250828874700885773307846$ \\
XANS $=1.474989038025216081528867562$ & $\mathrm{XANS}=-0.686026232904809407653345943$ \\
XANS $=1.474989038334796698226369926$ & XANS $=-0.686002934602659308893635171$ \\
XANS $=1.474989038334796698226369926$ & XANS $=-0.686002948238860277285766642$ \\
1.47498903833480 & XANS $=-0.686002948238860088547852456$ \\
$-1.7763568394003 \mathrm{~d}-15$ & -0.68600294823886 \\
& $-1.1102230246252 \mathrm{~d}-16$ \\
&
\end{tabular}

(Table b)

\section{\$4. Conclusion}

New Muller's Method is different from Muller's Method in the following points:

1) New Muller's Method starts from two initial approximations $X_{0}, X_{1}$ and $X_{2}=\left(X_{0}+X_{1}\right) / 2$ is used as an intermediate initial approximation.

2) As being shown at the Table $\mathbb{b}$, when the initial approximation was not almost the approached value of the root we failed. But $\mathbb{N e w}$ Muller's Method is a otherwise. (By the intermediate value theorem, it is explicit.) 


\section{References}

[1] Muller, D. E., A method for solving algebraic equation using an automatic computer, Mathematical Tables and other Aids to Computation, No. 10, (1956), 208-215.

[2] Richard, D. L. and Burden, J., Douglas Faires Numerical Analysis third edition, Wadsworth, Inc., 1984, 66-77.

[3] Kadlanskv, I., Set theory and Metric space, Chelsea Publishing Company, 1977. 